\title{
Dancing the digital age: a survey of the new technologies in the choreographic process
}

\author{
Gonzalo Preciado-Azanza \\ The Latvian National Ballet \\ Dr. Adesola Akinleye \\ Middlesex University
}

\begin{abstract}
This article considers fifty-eight selected dance works created during the time period of 2000-2018. In doing so the work of renown artists Wayne McGregor, Garry Stewart, Dawn Stopiello and Bill T. Jones have been used as case studies to highlight how the eminence of these choreographers has engaged dance as a meeting point and merging point for humanity and 'New technology'. The article reviews the impact of new technologies as an essential tool in the creative processes of dance and exploration of the moving-body. Innovative technologies in the 21st Century have offered choreographers new capacities for the creation of movement. These explorations into the performance space advance insights into broader questions of the human body at the intersection of arts and science. The choreographers' exploration of the dancing form cultivates questions about how the human body extends, begins, ends and is present. As the digital age proposes new ways to (re) imagine the communication and impact of the human body we suggest these artistic collaborations also offer insights into commonalities and places of exchange across notions of art versus science. These choreographers inter-disciplinary artistic endeavors, into how the moving body transacts and is harnessed as a mode of expression reveal deeper possibilities of the ontology of the lived-experience.
\end{abstract}

Keywords: Choreography, Collaboration, Dance, Digital Age, New Technology, Place 
The question of where the human body ends and begins is renegotiated through the interplay of choreographic work engaging with new technology. Dance movement comes from an intension or expression that creates corporeal repercussions in the physicality of the dancer. However, dance also goes beyond the dancer's skin, engaging with the assemblage that is the dance costume, stage, video, code, projection even engaging with the body of the computer operator or lighting technician. Performers move the spaces, lighting, and aesthetic of the performance as much as they move the muscle and bone of their physical body. As choreographers engage with the creative process, new technology connects us in barely explored ways across geographic and temporal plains only imagined as traversable a few years ago. During these kinds of collaborations between choreographer and technologists the performer exemplifies the provocative concept of the Body Without Organ (Deleuze \& Guattari, 1983, 1987) originally introduced in the fields of philosopher (Gilles Deleuze) and psychoanalyst (Félix Guattari) (Buchanan, 2010). Dance moves within, through, and beyond the skin of the dancer as the form creates shapes, rhythms, and relationships. Following the 20th Century pioneering work of artists such as eminent choreographers Merce Cunningham (Brown, 2007; Cunningham et al., 1998) and William Forsythe (Respini et al., 2018), the 21st Century technology and the internet bring further opportunity for choreographers to explore how, where, and when dance can happen.

The mechanisms of how the moving body responds to change and is harnessed as a mode of expression to reveal deeper understanding of the ontology of the lived-experience is the muse of both the artist and the scientist (Root-Bernstein, 1989). Although the intersection of art and science, that is the dancing body, have been long interwoven in Western philosophy, new technologies in sciences currently offer artists an absorbing scientific and technological creative playfulness (Wilson, 2010). The notion of creativity has been interwoven across artistic and scientific endeavors but the word itself has not existed in English until quite recently (Runco \& Jaeger, 2012). In dance, our understanding of creativity as makermaking is challenged and progressed by the digital age. Part of the revolution in the choreographic process that new technologies offer is the relationship performers have with audience. The experience of and literacy in new technologies that audiences bring to dance performances offers new collaborations and intersections between art, artist and onlooker. The role of audience is no-longer static. The audience becomes a part of the immediate creation of the art work in front of them. Audiences can be physically thousands of miles away or spread apart and yet through new technology, be connected in the moment of the dance 'happening. The choreographer has the ability to draw in or reference experiences, knowledges, and objects of our unconscious personal and collective everyday through interactions with images, actions, and sounds of personalized social media or personal technological devices. Thus, the dance performance is at once public and yet private. Throughout the last three decades, dance practices have witnessed a great acceleration through technological progress. This article reflects on how new technologies have been used in the work of four choreographers to pursue creative goals in 21st Century Professional Dance (Corazza 2016, pp. 263). In doing this, an initial effort has been made to map the lexis of ideas that the partnership of choreography and technologist can navigate.

\section{Manifestations of New technologies in contemporary choreography}

It would not be possible to survey across the many communities of dancers working on a global level. Choreographers working in USA, UK, and Australia were sampled. Therefore, these are the dance-cultural landscapes and sociocultural environments that are present in the research. To overview the field of dance's engagement with new technology across these locations, four case studies have been taken: Wayne 
McGregor in the United Kingdom, Dawn Stapiello and Bill T. Jones in the United States, and Garry Stewart in Australia. To analyze/explore the choreographers' employ of technology in dance creations we began by determining typologies. Thirteen categories of new technologies (see Table 1) were identified. Reflecting on the traditional expectation of the dance emanating from the dancers' body the thirteen categories in Table 1 were ordered as they extend the skin of the dancer's human body. That is costumes primarily being close to the tactile act of the body moving, while projection, for instance, has the possibility of being thousands of miles away from the physical body of the dancer it is interacting with. Of course, what is exciting about the possibilities of choreographing with new technologies is how this partnership can disrupt and challenge the limits of human activity, and dance happens beyond the dancer's body.

\section{Table 1}

Classification of New Technologies used in the selected choreographies

\begin{tabular}{|c|c|c|c|c|}
\hline $\begin{array}{l}\text { Relationship } \\
\text { with the Body }\end{array}$ & \multicolumn{2}{|c|}{ New Technology } & Description & $\begin{array}{l}\text { Examples of choreographies } \\
\text { where it had been used }\end{array}$ \\
\hline \multirow{2}{*}{ Nearby } & $C D$ & $\begin{array}{l}\text { Costume } \\
\text { Design }\end{array}$ & $\begin{array}{l}\text { The creation and integration of lights into the } \\
\text { piece to develop further its atmosphere. It } \\
\text { has been used in the majority of the works } \\
\text { to enhance certain aspects of the dancer's } \\
\text { body for a better development of the chore- } \\
\text { ography }\end{array}$ & $\begin{array}{l}\text { The majority of the choreogra- } \\
\text { phies by Bill T. Jones such as } \\
\text { Black Suzanne (Jones, 2002), as } \\
\text { well as in Dyad } 1909 \text { (McGregor, } \\
\text { 2009) \& MOVEment (McGregor, } \\
\text { 2015) }\end{array}$ \\
\hline & ME & $\begin{array}{l}\text { Mechanical } \\
\text { Extensions }\end{array}$ & $\begin{array}{l}\text { The artificial tools and artefacts designed to } \\
\text { increase the adaptation of our specie to the } \\
\text { environment that surround us. It has been } \\
\text { used in Nemesis to enhance the choreo- } \\
\text { graphic vocabulary of the dancer's limbs. }\end{array}$ & Nemesis (McGregor, 2001) \\
\hline \multirow{3}{*}{ Median } & LD & $\begin{array}{l}\text { Lighting } \\
\text { Design }\end{array}$ & $\begin{array}{l}\text { The creation and integration of lights into } \\
\text { the piece to develop further its atmosphere. } \\
\text { It has been used in all works to enhance cer- } \\
\text { tain choreographic aspects }\end{array}$ & $\begin{array}{l}\text { It has been used in all the cho- } \\
\text { reographies analysed such as } \\
\text { Tree of Codes (McGregor, 2015) } \\
\text { Multiverse (Stewart, 2014), Loop- } \\
\text { diver (Stopiello, 2009) \& Chapel/ } \\
\text { Chapter (Jones, 2006) }\end{array}$ \\
\hline & VD & $\begin{array}{l}\text { Video } \\
\text { Design }\end{array}$ & $\begin{array}{l}\text { The creation and integration of film and } \\
\text { motion graphics into the piece. It has been } \\
\text { used in the majority of the works to enhance } \\
\text { certain choreographic aspects }\end{array}$ & $\begin{array}{l}\text { The majority of the choreogra- } \\
\text { phies such as Tree of Codes (Mc- } \\
\text { Gregor, 2015) Multiverse (Stewart, } \\
\text { 2014), Loopdiver (Stopiello, 2009) } \\
\text { \& } \\
\text { Chapel/Chapter (Jones, 2006) }\end{array}$ \\
\hline & SoD & $\begin{array}{l}\text { Sound } \\
\text { Design }\end{array}$ & $\begin{array}{l}\text { The creation and integration of sounds into } \\
\text { the piece It has been used in the several } \\
\text { Jones's works such as A Quarreling Pair as } \\
\text { a complement to music }\end{array}$ & A Quarreling Pair (Jones, 2007) \\
\hline
\end{tabular}




\begin{tabular}{|c|c|c|c|c|}
\hline $\begin{array}{l}\text { Relationship } \\
\text { with the Body } \\
\text { (Continued) }\end{array}$ & \multicolumn{2}{|c|}{ New Technology } & Description & $\begin{array}{l}\text { Examples of choreographies } \\
\text { where it had been used }\end{array}$ \\
\hline \multirow{2}{*}{ Distant } & SD & Set Design & $\begin{array}{l}\text { The creation of the theatrical scenery to sup- } \\
\text { port the artistic goals of the production. It } \\
\text { has been used in the majority of the work as } \\
\text { an essential element of it and even as part of } \\
\text { the choreography as well }\end{array}$ & $\begin{array}{l}\text { The majority of the choreogra- } \\
\text { phies such as Tree of Codes (Mc- } \\
\text { Gregor, 2015) Multiverse (Stewart, } \\
\text { 2014), Loopdiver (Stopiello, 2009) } \\
\text { \& } \\
\text { Chapel/Chapter (Jones, 2006) }\end{array}$ \\
\hline & $\begin{array}{l}\text { 3D- } \\
\text { MS }\end{array}$ & $\begin{array}{l}\text { 3D Moving } \\
\text { Set }\end{array}$ & $\begin{array}{l}\text { A three dimensional set design that has been } \\
\text { created to move in certain pre-established } \\
\text { parts of the stage. It has been used in Entity } \\
\text { both as part of the set and the choreography } \\
\text { as well, since the dancers interact with them } \\
\text { throughout the work }\end{array}$ & Entity (McGregor, 2008) \\
\hline \multirow{6}{*}{ Distant } & Rob & Robotics & $\begin{array}{l}\text { The technologies that are used to develop } \\
\text { machines that can substitute for humans } \\
\text { and replicate human actions. It has been } \\
\text { used in Devolution to perform certain } \\
\text { choreographic aspects that could not be } \\
\text { performed by the dancers themselves }\end{array}$ & Devolution (Stewart, 2006) \\
\hline & $\begin{array}{l}\text { 3D- } \\
\text { CTI }\end{array}$ & $\begin{array}{l}\text { 3D Creative } \\
\text { Informatic } \\
\text { Tool }\end{array}$ & $\begin{array}{l}\text { A computer program designed to develop } \\
\text { and create three dimensional products, } \\
\text { following some pre-established guidelines. } \\
\text { It has been used in Atomos as a source of } \\
\text { inspiration for the creation of the movement } \\
\text { by the dancers }\end{array}$ & Atomos (McGregor, 2013) \\
\hline & $3 D-G$ & $\begin{array}{l}\text { 3D Graph- } \\
\text { ics }\end{array}$ & $\begin{array}{l}\text { Graphics that use a three dimensional } \\
\text { representation of geometric data to perform } \\
\text { calculations and to render 2D images. It has } \\
\text { been used in Multiverse both as part of the } \\
\text { set and the choreography as well, since the } \\
\text { dancers interact with them throughout the } \\
\text { work }\end{array}$ & Multiverse (Stewart, 2014) \\
\hline & LI & $\begin{array}{l}\text { Light } \\
\text { Installation }\end{array}$ & $\begin{array}{l}\text { An applied art form in which light is the main } \\
\text { medium of expression in which a sculpture } \\
\text { produces light or vice versa. It has been } \\
\text { used in Future Self as an essential element } \\
\text { of this work, in fact as another performer } \\
\text { more }\end{array}$ & Future Self (McGregor, 2012) \\
\hline & Al & $\begin{array}{l}\text { Art } \\
\text { Installation }\end{array}$ & $\begin{array}{l}\text { An artistic genre of three dimensional works } \\
\text { that often are site-specific and designed } \\
\text { to transform the perception of a space. It } \\
\text { has been used in Azimuth as an essential } \\
\text { element of this work, in fact as another } \\
\text { performer more }\end{array}$ & Azimuth (McGregor, 2013) \\
\hline & $\mathrm{Ml}$ & $\begin{array}{l}\text { Multimedia } \\
\text { Installation }\end{array}$ & $\begin{array}{l}\text { A variant of an Art Installation that uses the } \\
\text { multimedia technologies as its main medium } \\
\text { of expression. It will be used in Swarn as } \\
\text { an essential element of this work, in fact as } \\
\text { another performer more }\end{array}$ & $\begin{array}{l}\text { Swarn (Under development) } \\
\text { (Stopiello, 2018) }\end{array}$ \\
\hline
\end{tabular}


The intension of movement, its repercussions and its effect all inhabit the physical body of the dancers, but also create, change, and respond to the aesthetic, temporality, exhibit, and position of the performance. The muscle and bone of the physical dancing body and the code, or mechanics of the technology bring opportunity for choreographers to explore how, where and when dance can happen. For instance, costume design $(\mathrm{CD})$ has been used remarkably by Bill $\mathrm{T}$. Jones in fourteen choreographies to enhance certain aspects of the dancer's body, whilst mechanical extensions (ME) have been used by Wayne McGregor in Nemesis (2001) as an augmentation of the choreographic vocabulary of the dancers' limbs. In contrast, the more 'distant' from the physical body, new technologies such as robotics (Rob), 3D graphics (3D-G) create installations that become environments for dance to happen, such as the ones in Devolution (2006) and The Beginning of Nature (2018) by Garry Stewart. An attempt was made to quantify the frequency and variety of the new technologies to have an idea of the impact of technological advances over the two past decades. Fifty-eight choreographies were analyzed: twenty made by Wayne McGregor, ten by Garry Stewart, eight by Dawn Stopiello, and twenty by Bill $\mathrm{T}$. Jones (see Table 2).

\section{Table 2}

Dance creations made in the XXI century included in the analysis

\begin{tabular}{|c|c|c|c|c|c|}
\hline Abbreviation & Title & Choreographer & Company & City \& Country & Year \\
\hline $\begin{array}{l}\text { Stopiello } \\
\text { (Rosenkruetz) }\end{array}$ & $\begin{array}{l}\text { The chemical } \\
\text { wedding of Chris- } \\
\text { tian Rosenkruetz }\end{array}$ & Dawn Stopiello & Troika Ranch & Lincoln (USA) & 2000 \\
\hline Stopiello (DEVO) & SUITE DEVO & Dawn Stopiello & Troika Ranch & New York (USA) & 2001 \\
\hline Stopiello (Rien) & Reine Rien & Dawn Stopiello & Troika Ranch & New York (USA) & 2001 \\
\hline $\begin{array}{l}\text { Stopiello } \\
\text { (Memory) }\end{array}$ & Future of Memory & Dawn Stopiello & Troika Ranch & New York (USA) & 2003 \\
\hline $\begin{array}{l}\text { Stopiello } \\
\text { (Surfacing) }\end{array}$ & Surfacing & Dawn Stopiello & Troika Ranch & New York (USA) & 2004 \\
\hline $\begin{array}{l}\text { Stopiello } \\
\text { ([R]evolutions) }\end{array}$ & 16 [R]evolutions & Dawn Stopiello & Troika Ranch & New York (USA) & 2006 \\
\hline $\begin{array}{l}\text { Stopiello } \\
\text { (Loopdiver) }\end{array}$ & Loopdiver & Dawn Stopiello & Troika Ranch & Lincoln (USA) & 2009 \\
\hline Stopiello (Swarm) & $\begin{array}{l}\text { Swarm } \\
\text { (Under develop- } \\
\text { ment) }\end{array}$ & Dawn Stopiello & Troika Ranch & - & 2018 \\
\hline $\begin{array}{l}\text { Stewart } \\
\text { (Birdbrain) }\end{array}$ & Birdbrain & Garry Stewart & Australian Dance Theatre & $\begin{array}{l}\text { Adelaide (Aus- } \\
\text { tralia) }\end{array}$ & 2000 \\
\hline Stewart (Unbeauty) & $\begin{array}{l}\text { The Age of Un- } \\
\text { beauty }\end{array}$ & Garry Stewart & Australian Dance Theatre & $\begin{array}{l}\text { Adelaide (Aus- } \\
\text { tralia) }\end{array}$ & 2002 \\
\hline Stewart (HELD) & HELD & Garry Stewart & Australian Dance Theatre & $\begin{array}{l}\text { Adelaide (Aus- } \\
\text { tralia) }\end{array}$ & 2004 \\
\hline Stewart (Nascent) & Nascent & Garry Stewart & Australian Dance Theatre & $\begin{array}{l}\text { Adelaide (Aus- } \\
\text { tralia) }\end{array}$ & 2005 \\
\hline
\end{tabular}




\begin{tabular}{|c|c|c|c|c|c|}
\hline $\begin{array}{l}\text { Abbreviation } \\
\text { (Continued) }\end{array}$ & Title & Choreographer & Company & City \& Country & Year \\
\hline Stewart (Birdbrain) & Devolution & Garry Stewart & Australian Dance Theatre & $\begin{array}{l}\text { Adelaide (Aus- } \\
\text { tralia) }\end{array}$ & 2006 \\
\hline $\begin{array}{l}\text { Stewart (Devolu- } \\
\text { tion) }\end{array}$ & Worldhood & Garry Stewart & Australian Dance Theatre & $\begin{array}{l}\text { Adelaide (Aus- } \\
\text { tralia) }\end{array}$ & 2011 \\
\hline Stewart (Self) & Be Your Self & Garry Stewart & Australian Dance Theatre & $\begin{array}{l}\text { Adelaide (Aus- } \\
\text { tralia) }\end{array}$ & 2013 \\
\hline Stewart (Proximity) & Proximity & Garry Stewart & Australian Dance Theatre & $\begin{array}{l}\text { Adelaide (Aus- } \\
\text { tralia) }\end{array}$ & 2013 \\
\hline $\begin{array}{l}\text { Stewart (Multi- } \\
\text { verse) }\end{array}$ & Multiverse & Garry Stewart & Australian Dance Theatre & $\begin{array}{l}\text { Adelaide (Aus- } \\
\text { tralia) }\end{array}$ & 2014 \\
\hline Stewart (Nature) & $\begin{array}{l}\text { The Beginning of } \\
\text { Nature }\end{array}$ & Garry Stewart & Australian Dance Theatre & $\begin{array}{l}\text { Adelaide (Aus- } \\
\text { tralia) }\end{array}$ & 2018 \\
\hline McGregor (Aeon) & Aeon & Wayne McGregor & Studio Wayne McGregor & London (UK) & 2000 \\
\hline $\begin{array}{l}\text { McGregor (Nem- } \\
\text { esis) }\end{array}$ & Nemesis & Wayne McGregor & Studio Wayne McGregor & London (UK) & 2001 \\
\hline $\begin{array}{l}\text { McGregor (Se- } \\
\text { quences) }\end{array}$ & Polar Sequences & Wayne McGregor & Studio Wayne McGregor & London (UK) & 2003 \\
\hline McGregor (AtaXia) & AtaXia & Wayne McGregor & Studio Wayne McGregor & London (UK) & 2004 \\
\hline McGregor (Amu) & Amu & Wayne McGregor & Studio Wayne McGregor & London (UK) & 2005 \\
\hline McGregor (Erazor) & Erazor & Wayne McGregor & Studio Wayne McGregor & London (UK) & 2006 \\
\hline McGregor (Entity) & Entity & Wayne McGregor & Studio Wayne McGregor & London (UK) & 2008 \\
\hline McGregor (1909) & Dyad 1909 & Wayne McGregor & Studio Wayne McGregor & London (UK) & 2009 \\
\hline McGregor (FAR) & FAR & Wayne McGregor & Studio Wayne McGregor & London (UK) & 2010 \\
\hline $\begin{array}{l}\text { McGregor (UN- } \\
\text { DANCE) }\end{array}$ & UNDANCE & Wayne McGregor & Studio Wayne McGregor & London (UK) & 2011 \\
\hline McGregor (Self) & Future Self & Wayne McGregor & Studio Wayne McGregor & Berlin (Germany) & 2012 \\
\hline McGregor (Room) & Rain Room & Wayne McGregor & Studio Wayne McGregor & London (UK) & 2012 \\
\hline $\begin{array}{l}\text { McGregor (Az- } \\
\text { imtuh) }\end{array}$ & Azimuth & Wayne McGregor & Studio Wayne McGregor & London (UK) & 2013 \\
\hline $\begin{array}{l}\text { McGregor (Ato- } \\
\text { mos) }\end{array}$ & Atomos & Wayne McGregor & Studio Wayne McGregor & London (UK) & 2013 \\
\hline $\begin{array}{l}\text { McGregor (MOVE- } \\
\text { ment) }\end{array}$ & MOVEment & Wayne McGregor & Studio Wayne McGregor & London (UK) & 2015 \\
\hline
\end{tabular}




\begin{tabular}{|c|c|c|c|c|c|}
\hline $\begin{array}{l}\text { Abbreviation } \\
\text { (Continued) }\end{array}$ & Title & Choreographer & Company & City \& Country & Year \\
\hline McGregor (Codes) & Tree of Codes & Wayne McGregor & Studio Wayne McGregor & London (UK) & 2015 \\
\hline $\begin{array}{l}\text { McGregor (Autobi- } \\
\text { ography) }\end{array}$ & Autobiography & Wayne McGregor & Studio Wayne McGregor & London (UK) & 2017 \\
\hline $\begin{array}{l}\text { McGregor (Collec- } \\
\text { tion) }\end{array}$ & $\begin{array}{l}\text { In Residence: } \\
\text { Zabludowicz } \\
\text { Collection }\end{array}$ & Wayne McGregor & Studio Wayne McGregor & London (UK) & 2017 \\
\hline McGregor (Case) & $\begin{array}{l}\text { Winged Bull in the } \\
\text { Elephant Case }\end{array}$ & Wayne McGregor & Studio Wayne McGregor & London (UK) & 2018 \\
\hline McGregor (Forms) & Bach Forrms & Wayne McGregor & Studio Wayne McGregor & London (UK) & 2018 \\
\hline Jones (Project) & The Table Project & Bill T. Jones & $\begin{array}{l}\text { Bill T. Jones Arnie Zane } \\
\text { Dance Company }\end{array}$ & $\begin{array}{l}\text { Minneapolis } \\
\text { (USA) }\end{array}$ & 2001 \\
\hline Jones (Power/Full) & Power/Full & Bill T. Jones & $\begin{array}{l}\text { Bill T. Jones Arnie Zane } \\
\text { Dance Company }\end{array}$ & Ravenna (Italy) & 2002 \\
\hline Jones (Were) & There Were... & Bill T. Jones & $\begin{array}{l}\text { Bill T. Jones Arnie Zane } \\
\text { Dance Company }\end{array}$ & - & 2002 \\
\hline Jones (Kurtag) & $\begin{array}{l}\text { World II (18 } \\
\text { Movements to } \\
\text { Kurtag) }\end{array}$ & Bill T. Jones & $\begin{array}{l}\text { Bill T. Jones Arnie Zane } \\
\text { Dance Company }\end{array}$ & Lee (USA) & 2002 \\
\hline Jones (Suzanne) & Black Suzanne & Bill T. Jones & $\begin{array}{l}\text { Bill T. Jones Arnie Zane } \\
\text { Dance Company }\end{array}$ & lowa (USA) & 2002 \\
\hline $\begin{array}{l}\text { Jones (WITHOUT/ } \\
\text { IN) }\end{array}$ & $\begin{array}{l}\text { WORLD WITH- } \\
\text { OUT/IN }\end{array}$ & Bill T. Jones & $\begin{array}{l}\text { Bill T. Jones Arnie Zane } \\
\text { Dance Company }\end{array}$ & lowa (USA) & 2002 \\
\hline Jones (Verbum) & Verbum & Bill T. Jones & $\begin{array}{l}\text { Bill T. Jones Arnie Zane } \\
\text { Dance Company }\end{array}$ & lowa (USA) & 2002 \\
\hline Jones (Circle) & $\begin{array}{l}\text { Mercy } 10 \times 8 \text { on a } \\
\text { Circle }\end{array}$ & Bill T. Jones & $\begin{array}{l}\text { Bill T. Jones Arnie Zane } \\
\text { Dance Company }\end{array}$ & $\begin{array}{l}\text { New York City } \\
\text { (USA) }\end{array}$ & 2003 \\
\hline Jones (Evening) & Another Evening & Bill T. Jones & $\begin{array}{l}\text { Bill T. Jones Arnie Zane } \\
\text { Dance Company }\end{array}$ & $\begin{array}{l}\text { New York City } \\
\text { (USA) }\end{array}$ & 2003 \\
\hline Jones (Saying) & As I Was Saying & Bill T. Jones & $\begin{array}{l}\text { Bill T. Jones Arnie Zane } \\
\text { Dance Company }\end{array}$ & $\begin{array}{l}\text { Minneapolis } \\
\text { (USA) }\end{array}$ & 2005 \\
\hline Jones (Date) & Blind Date & Bill T. Jones & $\begin{array}{l}\text { Bill T. Jones Arnie Zane } \\
\text { Dance Company }\end{array}$ & $\begin{array}{l}\text { New York City } \\
\text { (USA) }\end{array}$ & 2005 \\
\hline Jones (Down) & $\begin{array}{l}\text { Another Evening: I } \\
\text { Bow Down }\end{array}$ & Bill T. Jones & $\begin{array}{l}\text { Bill T. Jones Arnie Zane } \\
\text { Dance Company }\end{array}$ & $\begin{array}{l}\text { New York City } \\
\text { (USA) }\end{array}$ & 2006 \\
\hline $\begin{array}{l}\text { Jones (Chapel/ } \\
\text { Chapter) }\end{array}$ & Chapel/Chapter & Bill T. Jones & $\begin{array}{l}\text { Bill T. Jones Arnie Zane } \\
\text { Dance Company }\end{array}$ & $\begin{array}{l}\text { New York City } \\
\text { (USA) }\end{array}$ & 2006 \\
\hline Jones (Pair) & A Quarreling Pair & Bill T. Jones & $\begin{array}{l}\text { Bill T. Jones Arnie Zane } \\
\text { Dance Company }\end{array}$ & Montclair (USA) & 2007 \\
\hline Jones (Migrations) & 100 Migrations & Bill T. Jones & $\begin{array}{l}\text { Bill T. Jones Arnie Zane } \\
\text { Dance Company }\end{array}$ & Virginia (USA) & 2008 \\
\hline
\end{tabular}




\begin{tabular}{|c|c|c|c|c|c|}
\hline $\begin{array}{l}\text { Abbreviation } \\
\text { (Continued) }\end{array}$ & Title & Choreographer & Company & City \& Country & Year \\
\hline Jones (Proposition) & $\begin{array}{l}\text { Serenade/The } \\
\text { Proposition }\end{array}$ & Bill T. Jones & $\begin{array}{l}\text { Bill T. Jones Arnie Zane } \\
\text { Dance Company }\end{array}$ & Durham (USA) & 2008 \\
\hline Jones (Pray) & $\begin{array}{l}\text { Fondly Do We } \\
\text { Hope... Fervently } \\
\text { Do We Pray }\end{array}$ & Bill T. Jones & $\begin{array}{l}\text { Bill T. Jones Arnie Zane } \\
\text { Dance Company }\end{array}$ & $\begin{array}{l}\text { Highland Parks } \\
\text { (USA) }\end{array}$ & 2009 \\
\hline $\begin{array}{l}\text { Jones (Venice/Ar- } \\
\text { senale) }\end{array}$ & $\begin{array}{l}\text { Another Evening: } \\
\text { Venice/Arsenale }\end{array}$ & Bill T. Jones & $\begin{array}{l}\text { Bill T. Jones Arnie Zane } \\
\text { Dance Company }\end{array}$ & Venice (Italy) & 2010 \\
\hline Jones (Story/Time) & Story/Time & Bill T. Jones & $\begin{array}{l}\text { Bill T. Jones Arnie Zane } \\
\text { Dance Company }\end{array}$ & Montclair (USA) & 2014 \\
\hline Jones (Nephew) & $\begin{array}{l}\text { A Letter to my } \\
\text { Nephew }\end{array}$ & Bill T. Jones & $\begin{array}{l}\text { Bill T. Jones Arnie Zane } \\
\text { Dance Company }\end{array}$ & $\begin{array}{l}\text { New York City } \\
\text { (USA) }\end{array}$ & 2017 \\
\hline
\end{tabular}

The focus was on works these choreographers made for their own companies. The study period was from 2000 to 2018, during this period all four choreographers also guest composed works for international companies beyond their own. Therefore, this survey of fifty-eight works is possibly representative of creative processes engaged with by professional dance companies more generally during this period. Video documentation was used, photographs of performances, and reviews of the four choreographers web-sites. The survey scheme was revised a few times to obtain empirical data as detailed as possible. (Table 3 compiles the new technologies used within each choreography. While Table 4 overviews use of new technologies by year.)

\section{Table 3}

Survey of the new technologies used in the analyzed dance creations made in the XXI century among the cases of study. Abbreviations according to Table 2, the order follows the new technologies from most to least used

\begin{tabular}{|c|c|c|c|c|c|c|c|c|c|c|c|c|c|c|}
\hline Choreography & LD & SD & VD & CD & SoD & Rob & ME & $\begin{array}{l}\text { 3D- } \\
\text { MS }\end{array}$ & $\begin{array}{l}\text { 3D- } \\
\text { CIT }\end{array}$ & $3 D-G$ & LI & $\mathrm{Al}$ & $\mathrm{Ml}$ & Total \\
\hline $\begin{array}{l}\text { Stopiello } \\
\text { (Rosenkruetz) }\end{array}$ & $x$ & & $x$ & & & & & & & & & & & 2 \\
\hline $\begin{array}{l}\text { Stopiello } \\
\text { (DEVO) }\end{array}$ & $x$ & & $x$ & & & & & & & & & & & 2 \\
\hline Stopiello (Rien) & $x$ & & $x$ & & & & & & & & & & & 2 \\
\hline $\begin{array}{l}\text { Stopiello } \\
\text { (Memory) }\end{array}$ & $x$ & $x$ & $x$ & & & & & & & & & & & 3 \\
\hline $\begin{array}{l}\text { Stopiello } \\
\text { (Surfacing) }\end{array}$ & $x$ & $x$ & $x$ & & & & & & & & & & & 3 \\
\hline $\begin{array}{l}\text { Stopiello } \\
\text { ([R]evolutions) }\end{array}$ & $x$ & $x$ & $x$ & & & & & & & & & & & 3 \\
\hline $\begin{array}{l}\text { Stopiello } \\
\text { (Loopdiver) }\end{array}$ & $x$ & $x$ & $x$ & & & & & & & & & & & 3 \\
\hline $\begin{array}{l}\text { Stopiello } \\
\text { (Swarm) }\end{array}$ & $x$ & $x$ & $x$ & & & & & & & & & & $x$ & 4 \\
\hline Dawn Stopiello & 8 & 5 & 8 & 0 & 0 & 0 & 0 & 0 & 0 & 0 & 0 & 0 & 1 & \\
\hline $\begin{array}{l}\text { Stewart } \\
\text { (Birdbrain) }\end{array}$ & $x$ & & $x$ & & & & & & & & & & & 2 \\
\hline $\begin{array}{l}\text { Stewart } \\
\text { (Unbeauty) }\end{array}$ & $x$ & $x$ & $x$ & & & & & & & & & & & 3 \\
\hline
\end{tabular}




\begin{tabular}{|c|c|c|c|c|c|c|c|c|c|c|c|c|c|c|}
\hline $\begin{array}{l}\text { Choreography } \\
\text { (Continued) }\end{array}$ & LD & SD & VD & CD & SoD & Rob & ME & $\begin{array}{l}\text { 3D- } \\
\text { MS }\end{array}$ & $\begin{array}{l}\text { 3D- } \\
\text { CIT }\end{array}$ & $3 D-G$ & $\mathrm{LI}$ & $\mathrm{Al}$ & MI & Total \\
\hline Stewart (HELD) & $x$ & & $x$ & & & & & $x$ & & & & & & 3 \\
\hline $\begin{array}{l}\text { Stewart } \\
\text { (Nascent) }\end{array}$ & $x$ & & $x$ & & & & & & & & & & & 2 \\
\hline $\begin{array}{l}\text { Stewart } \\
\text { (Birdbrain) }\end{array}$ & $x$ & $x$ & & & & $x$ & & & & & & & & 3 \\
\hline $\begin{array}{l}\text { Stewart } \\
\text { (Devolution) }\end{array}$ & $x$ & $x$ & $x$ & & & & & & & & & & & 3 \\
\hline Stewart (Self) & $x$ & $x$ & & & & & & & & & & & & 2 \\
\hline $\begin{array}{l}\text { Stewart } \\
\text { Proximity) }\end{array}$ & $x$ & $x$ & $x$ & & & & & & & & & & & 3 \\
\hline $\begin{array}{l}\text { Stewart } \\
\text { (Multiverse) }\end{array}$ & $x$ & $x$ & $x$ & & & & & & & $x$ & & & & 4 \\
\hline $\begin{array}{l}\text { Stewart } \\
\text { (Nature) }\end{array}$ & $x$ & & & & & & & & & & & & & 1 \\
\hline Garry Stewart & 10 & 6 & 7 & 0 & 0 & 1 & 0 & 1 & 0 & 1 & 0 & 0 & 0 & \\
\hline $\begin{array}{l}\text { McGregor } \\
\text { (Aeon) }\end{array}$ & $x$ & & $x$ & & & & & & & & & & & 2 \\
\hline $\begin{array}{l}\text { McGregor } \\
\text { (Nemesis) }\end{array}$ & $x$ & & $x$ & & & & $x$ & & & & & & & 3 \\
\hline $\begin{array}{l}\text { McGregor (Se- } \\
\text { quences) }\end{array}$ & $x$ & $x$ & & & & & & & & & & & & 2 \\
\hline $\begin{array}{l}\text { McGregor } \\
\text { (AtaXia) }\end{array}$ & $x$ & $x$ & & & & & & & & & & & & 2 \\
\hline $\begin{array}{l}\text { McGregor } \\
(\mathrm{Amu})\end{array}$ & $x$ & & & & & & & & & & & & & 1 \\
\hline $\begin{array}{l}\text { McGregor } \\
\text { (Erazor) }\end{array}$ & $x$ & $x$ & $x$ & & & & & & & & & & & 3 \\
\hline $\begin{array}{l}\text { McGregor } \\
\text { (Entity) }\end{array}$ & $x$ & & $x$ & & & & & $x$ & & & & & & 3 \\
\hline $\begin{array}{l}\text { McGregor } \\
(1909)\end{array}$ & $x$ & & $x$ & $x$ & & & & & & & & & & 3 \\
\hline $\begin{array}{l}\text { McGregor } \\
\text { (FAR) }\end{array}$ & $x$ & $x$ & & & & & & & & & & & & 2 \\
\hline $\begin{array}{l}\text { McGregor (UN- } \\
\text { DANCE) }\end{array}$ & $x$ & & $x$ & & & & & & & & & & & 2 \\
\hline McGregor (Self) & $x$ & $x$ & & & & & & & & & $x$ & & & 3 \\
\hline $\begin{array}{l}\text { McGregor } \\
\text { (Room) }\end{array}$ & $x$ & & & & & & & & & & & $x$ & & 2 \\
\hline $\begin{array}{l}\text { McGregor (Az- } \\
\text { imtuh) }\end{array}$ & $x$ & $x$ & & & & & & & & & & $x$ & & 3 \\
\hline $\begin{array}{l}\text { McGregor } \\
\text { (Atomos) }\end{array}$ & $x$ & $x$ & & & & & & & $x$ & & & & & 3 \\
\hline $\begin{array}{l}\text { McGregor } \\
\text { (MOVEment) }\end{array}$ & $x$ & & & $x$ & & & & & & & & & & 2 \\
\hline $\begin{array}{l}\text { McGregor } \\
\text { (Codes) }\end{array}$ & $x$ & $x$ & $x$ & & & & & & & & & & & 3 \\
\hline $\begin{array}{l}\text { McGregor (Au- } \\
\text { tobiography) }\end{array}$ & $x$ & & $x$ & & & & & & & & & & & 2 \\
\hline $\begin{array}{l}\text { McGregor (Col- } \\
\text { lection) }\end{array}$ & $x$ & $x$ & & & & & & & & & & & & 2 \\
\hline $\begin{array}{l}\text { McGregor } \\
\text { (Case) }\end{array}$ & $x$ & & $x$ & & & & & & & & & & & 2 \\
\hline
\end{tabular}




\begin{tabular}{|c|c|c|c|c|c|c|c|c|c|c|c|c|c|c|}
\hline $\begin{array}{l}\text { Choreography } \\
\text { (Continued) }\end{array}$ & LD & SD & VD & CD & SoD & Rob & ME & $\begin{array}{l}\text { 3D- } \\
\text { MS }\end{array}$ & $\begin{array}{l}\text { 3D- } \\
\text { CIT }\end{array}$ & $3 D-G$ & LI & $\mathrm{Al}$ & MI & Total \\
\hline $\begin{array}{l}\text { McGregor } \\
\text { (Forms) }\end{array}$ & $x$ & $x$ & & $x$ & & & & & & & & & & 3 \\
\hline $\begin{array}{l}\text { Wayne Mcgre- } \\
\text { gor }\end{array}$ & 20 & 10 & 9 & 3 & 0 & 0 & 1 & 1 & 1 & 0 & 1 & 2 & 0 & \\
\hline Jones (Project) & $x$ & $x$ & & & & & & & & & & & & 2 \\
\hline $\begin{array}{l}\text { Jones (Power/ } \\
\text { Full) }\end{array}$ & $x$ & & & & & & & & & & & & & 1 \\
\hline Jones (Were) & $x$ & $x$ & & $x$ & & & & & & & & & & 3 \\
\hline Jones (Kurtag) & $x$ & & & $x$ & & & & & & & & & & 2 \\
\hline $\begin{array}{l}\text { Jones } \\
\text { (Suzanne) }\end{array}$ & $x$ & $x$ & & $x$ & & & & & & & & & & 3 \\
\hline $\begin{array}{l}\text { Jones } \\
\text { (WITHOUT/IN) }\end{array}$ & $x$ & $x$ & & $x$ & & & & & & & & & & 3 \\
\hline $\begin{array}{l}\text { Jones } \\
\text { (Verbum) }\end{array}$ & $x$ & $x$ & & $x$ & & & & & & & & & & 3 \\
\hline Jones (Circle) & $x$ & & & $x$ & & & & & & & & & & 2 \\
\hline $\begin{array}{l}\text { Jones } \\
\text { (Evening) }\end{array}$ & $x$ & & & & & & & & & & & & & 1 \\
\hline $\begin{array}{l}\text { Jones } \\
\text { (Saying) }\end{array}$ & $x$ & $x$ & & $x$ & & & & & & & & & & 3 \\
\hline Jones (Date) & $x$ & $x$ & $x$ & $x$ & & & & & & & & & & 4 \\
\hline Jones (Down) & $x$ & $x$ & & $x$ & & & & & & & & & & 3 \\
\hline $\begin{array}{l}\text { Jones (Chapel/ } \\
\text { Chapter) }\end{array}$ & $x$ & $x$ & $x$ & & & & & & & & & & & 3 \\
\hline Jones (Pair) & $x$ & $x$ & $x$ & $x$ & $x$ & & & & & & & & & 5 \\
\hline $\begin{array}{l}\text { Jones } \\
\text { (Migrations) }\end{array}$ & $x$ & $x$ & & & $x$ & & & & & & & & & 3 \\
\hline $\begin{array}{l}\text { Jones } \\
\text { (Proposition) }\end{array}$ & $x$ & $x$ & $x$ & $x$ & $x$ & & & & & & & & & 5 \\
\hline Jones (Pray) & $x$ & & $x$ & $x$ & $x$ & & & & & & & & & 4 \\
\hline $\begin{array}{l}\text { Jones (Venice/ } \\
\text { Arsenale) }\end{array}$ & $x$ & $x$ & & $x$ & $x$ & & & & & & & & & 4 \\
\hline $\begin{array}{l}\text { Jones (Story/ } \\
\text { Time) }\end{array}$ & $x$ & $x$ & & $x$ & & & & & & & & & & 3 \\
\hline $\begin{array}{l}\text { Jones } \\
\text { (Nephew) }\end{array}$ & $x$ & & $x$ & & & & & & & & & & & 2 \\
\hline Bill T. Jones & 20 & 14 & 6 & 14 & 5 & 0 & 0 & 0 & 0 & 0 & 0 & 0 & 0 & \\
\hline
\end{tabular}

\section{Table 4}

Survey of the new technologies used in the analyzed dance creations made in the XXI century over the study period of time. The order follows the new technologies from most to least used

\begin{tabular}{|l|l|l|l|l|l|l|l|l|l|l|l|l|l|l|}
\hline Year & LD & SD & VD & CD & SoD & Rob & ME & $\begin{array}{l}\text { 3D- } \\
\text { MS }\end{array}$ & $\begin{array}{l}\text { 3D- } \\
\text { CIT }\end{array}$ & 3D-G & LI & Al & MI & Total \\
\hline 2000 & 3 & 0 & 3 & 0 & 0 & 0 & 0 & 0 & 0 & 0 & 0 & 0 & 0 & 6 \\
\hline 2001 & 4 & 1 & 3 & 0 & 0 & 0 & 1 & 0 & 0 & 0 & 0 & 0 & 0 & 9 \\
\hline
\end{tabular}




\begin{tabular}{|c|c|c|c|c|c|c|c|c|c|c|c|c|c|c|}
\hline $\begin{array}{l}\text { Chore- } \\
\text { ography } \\
\text { (Contin- } \\
\text { ued) }\end{array}$ & LD & SD & VD & CD & SoD & Rob & ME & $\begin{array}{l}\text { 3D- } \\
\text { MS }\end{array}$ & $\begin{array}{l}\text { 3D- } \\
\text { CIT }\end{array}$ & $3 D-G$ & LI & $\mathrm{Al}$ & $\mathrm{Ml}$ & Total \\
\hline 2002 & 7 & 5 & 1 & 5 & 0 & 0 & 0 & 0 & 0 & 0 & 0 & 0 & 0 & 18 \\
\hline 2003 & 4 & 2 & 1 & 1 & 0 & 0 & 0 & 0 & 0 & 0 & 0 & 0 & 0 & 8 \\
\hline 2004 & 3 & 2 & 2 & 0 & 0 & 0 & 0 & 1 & 0 & 0 & 0 & 0 & 0 & 8 \\
\hline 2005 & 4 & 2 & 2 & 2 & 0 & 0 & 0 & 0 & 0 & 0 & 0 & 0 & 0 & 10 \\
\hline 2006 & 5 & 5 & 3 & 1 & 0 & 1 & 0 & 0 & 0 & 0 & 0 & 0 & 0 & 15 \\
\hline 2007 & 1 & 1 & 1 & 1 & 1 & 0 & 0 & 0 & 0 & 0 & 0 & 0 & 0 & 5 \\
\hline 2008 & 3 & 2 & 2 & 1 & 2 & 0 & 0 & 1 & 0 & 0 & 0 & 0 & 0 & 11 \\
\hline 2009 & 3 & 1 & 3 & 2 & 1 & 0 & 0 & 0 & 0 & 0 & 0 & 0 & 0 & 10 \\
\hline 2010 & 2 & 2 & 0 & 1 & 1 & 0 & 0 & 0 & 0 & 0 & 0 & 0 & 0 & 6 \\
\hline 2011 & 2 & 1 & 2 & 0 & 0 & 0 & 0 & 0 & 0 & 0 & 0 & 0 & 0 & 5 \\
\hline 2012 & 2 & 1 & 0 & 0 & 0 & 0 & 0 & 0 & 0 & 0 & 1 & 1 & 0 & 5 \\
\hline 2013 & 4 & 4 & 1 & 0 & 0 & 0 & 0 & 0 & 1 & 0 & 0 & 1 & 0 & 11 \\
\hline 2014 & 2 & 2 & 1 & 1 & 0 & 0 & 0 & 0 & 0 & 1 & 0 & 0 & 0 & 7 \\
\hline 2015 & 2 & 1 & 1 & 1 & 0 & 0 & 0 & 0 & 0 & 0 & 0 & 0 & 0 & 5 \\
\hline 2016 & 0 & 0 & 0 & 0 & 0 & 0 & 0 & 0 & 0 & 0 & 0 & 0 & 0 & 0 \\
\hline 2017 & 3 & 1 & 2 & 0 & 0 & 0 & 0 & 0 & 0 & 0 & 0 & 0 & 0 & 6 \\
\hline 2018 & 4 & 2 & 2 & 1 & 0 & 0 & 0 & 0 & 0 & 0 & 0 & 0 & 1 & 10 \\
\hline Total & 58 & 35 & 30 & 16 & 5 & 1 & 1 & 2 & 1 & 1 & 1 & 2 & 1 & \\
\hline
\end{tabular}

\section{Reflections on the survey}

The survey process revealed the impact of new technologies in the creative process and exploration of the moving-body through choreography. The combination of new technology and dance embodied an ontological challenge to what it means to $\mathrm{Be}$ in Place (Akinleye, 2019). Where the dance/dancer ends and begins is a choreographic statement but also an orientation to describing Being-in-environment, as explored in pragmatism (Dewey \& Boydston, 2008) or phenomenology (Merleau-Ponty, 2002) ${ }^{1}$. We have
${ }^{1}$ It is beyond the scope of this article to discuss here however reflections drawn from the survey have indicated taken the ontological standpoint that the dancer's body is a structure that is porous to dance, thus dance happens within through and beyond the dancer's body (from a phenomenological perspective see MerleauPonty, 2002; from a pragmatist perspective see Dewey, 1958).

'...They live, that is, as much in processes across and "through" skins as in processes "within" skins.' (Dewey and Boydston, 2008 p. 119)

the direction for our own deliberation and choreographic exploration currently. 
If 'they' are the choreographies and 'skins' are of the dancers, Pragmatist John Dewey suggested the poetics of the dances we observed. The offer of Self beyond oneSelf becomes a fragility, strength and confrontation across the choreographies in the study, mirroring 21 st century questions of identity and networks of humanity that are global political challenges today.

Reflecting specifically on the technology used; light design (LD) is present in all the fifty-eight choreographies and has become an essential tool since Loie Fuller's pioneering Western creation of the Serpentine Dance (1891). Set design (SD) in thirty-five, as well as Video Design (VD) in thirty, are the new technologies most used, and therefore we considered these as key locations at this point in the development of the digital aesthetic on the dance staged space in the 21st century. However, the use of robotics (Rob) in Devolution (Stewart, 2006) or the 3D graphics (3D-G) in Multiverse (Stewart, 2014), indicate developments in how the dancer extends and is interactive out into the staged space. (see figure 1 - Bar charts of the Use of New Technologies used in the Dance creations made in the XXI century, a] By Cases of Study, b] By years through the study period of time. Abbreviations according to Table 2)

\section{Figure 1}

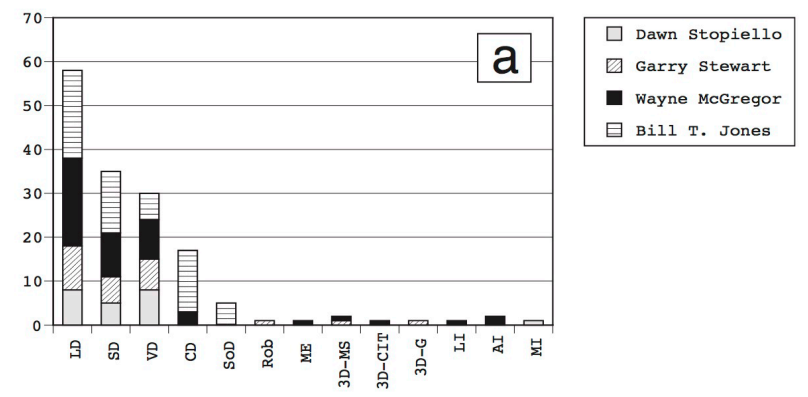

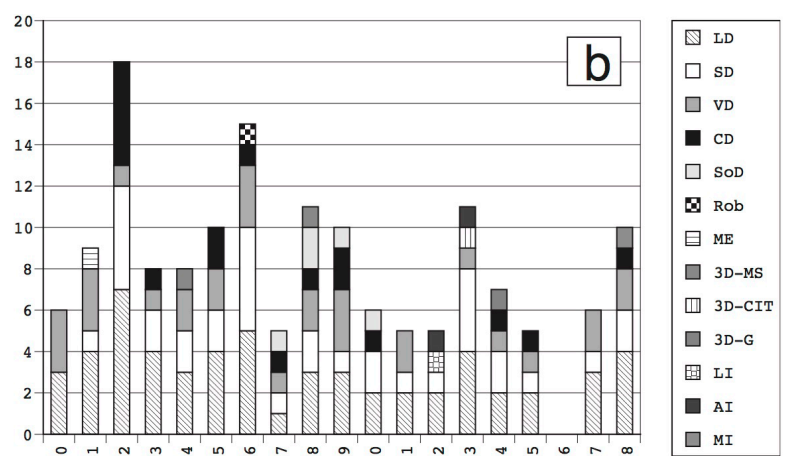

Eight pieces developed by Stopello between 2000 and 2018 were examined. Over this period, she has used four types of new technologies. Since 2003, LD, $\mathrm{SD}, \mathrm{VD}$, are present in all her choreographic works. However, her latest piece, Swarm, which Stopello's web-site describes as 'currently under development' (Troika Ranch, accessed 2019), includes a multimedia installation (MI). Stopello's creative process seems to be shaped by a progression in the use of new technologies, first two (LD, VD) between The chemical wedding of Christian Rosenkruetz (2000) and Reine Rien (2001) then three (LD, SD, VD) between Future of Memory (2003) and Loopdiver (2009), and currently four (LD, VD, SD, MI) in Swarm (2018).

Ten works created by Stewart over almost two decades (2000-2018) were viewed. Stewart used six from our typologies. He has been a pioneer in the use of robotics (Rob) in Devolution (2006), 3D moving set (3D-MS) in HELD (2002), which was used later also by McGregor (Entity, 2008) and 3D graphics (3D-G) in Multiverse $(2014)^{2}$, In terms of his choreographic process, Stewart, like Stopello, started with two different types (LD, VD in 2000), however since the opening of The Age of Unbeauty (2002), he began an intense research process, using an average of three new technologies for each piece. Unlike Stopiello, he has not had an upward progression of types of New Technology engagement.

\footnotetext{
${ }^{2}$ Multiverse is one of Stewart's most known pieces, having toured globally, with a great success both of critic and public (Australian Dance Theatre, 1999)
} 
For instance, he only uses one (LD) in his most recent work The Beginning of Nature (2018).

Twenty pieces from Wayne McGregor were examined. His work engaged with nine typologies (see Table 1). McGregor has created pioneering collaborations between dance and costume design (CD) in Dyad 1909 (2009), mechanical extensions (ME) in Nemesis (2001), 3D creative informatic tool (3D-CIT) in Atomos (2013), light installation (LI) in Future Self (2012) and art installation in Rain Room (2012). Nonetheless, the survey gives an impression that currently McGregor is less interested in exploring beyond the new technologies he used at the beginning of this decade, since from MOVEment (2015), he has been working with the same four new technologies alternately (LD, SD, VD, CD). In his last piece, Bach Forms (2018), McGregor incorporated the concept of the mathematical equations within The Art of Fugue of J.S. Bach, as McGregor considered it a stimulus for the human condition and therefore, a starting point on which to create this work (Studio Wayne McGregor, accessed 2019).

Bill T. Jones is a pioneering artist with a longevity of works dating back to 1970s. we looked at twenty pieces created between 2000 and 2018. It should be noted not all Jones choreographic creations use 'new technology', for this research there was a focus on the works where New Technology is used. Of Jones's works that were surveyed his key uses are LD, SD, VD, he has also used remarkable costume design (CD) in the majority of his pieces, as well as being a pioneer in the use of sound design (SoD) in A Quarreling Pair (2007). In Serenade/ The Proposition (2008) and A Quarreling Pair (2007), Jones used LD, SD, VD, CD. As with Stapiello and Stewart, Jones started using only two different types (LD, SD) in The Table Project (2001). He explored more typologies in his following works using an average of four new technologies for each piece. The most recent creation looked at by Jones, A Letter to my Nephew (2017), he has only used two (LD, VD).
The four artistshighlight that technologicaldevelopment can be seen as a fundamental motor of progress and stimuli in the geographical locations they create in. Technological inventions not only shape ways of life, but also change the mentality of millions of people, offering new understandings of what it is to be of the world. Dance is not disconnected from technological advances. Consequently, the type of transdisciplinary creative processes portrayed in the survey blur the lines between research and practice in the artistic process (Tornero, 2013). The development and exchange of ideas alongside these productive collaborations which bring scientists, engineers and artists together is reminiscent of Europe's Renaissance period. This was a time when what is to be human was redefined (Wynter, 2003), echoing ethical and ontological questions that new technologies solicit today. The artists' collaborations with laboratories such as the College of the Holy Cross in Worcester, Massachusetts (Stopello), the Motion Lab in Melbourne (Stewart) or the University of California in San Diego (McGregor) are tasked with how scientists and artists respond to the social political implications of creative knowledges, locations and resources as well as the performance art of originality and effectiveness (Runco \& Jaeger, 2012).

\section{Conclusion: New aesthetics \& New Technologies}

The great acceleration of scientific and technological progress in the 21st Century has permeated our society so deeply, that we have entered a new era: the digital era (Salter, 2010). Choreographers and dancers have played an essential role in the incorporation of new media such as software or interfaces on stage (Boerisch, 2006). The fifty-eight works in the survey illustrate that in the choreographic process the relationship between art and science is transactional. Creativity seems to make perceived borders between art and science permeable or even arbitrary. The genius of the choreographers in the study has made tangible, 
through their work, the idea that the creative process is the product of our technology and imagination (Tornero, 2013). What makes these dance artists influential today is that their work is seen as innovative and visionary. The eminence of these choreographers is that they are simultaneously innovator, researcher, and body - fabricating new technologies through the aesthetic and felt experience of moving in space. This art/science involves the discovery of humanness (Simó, 2013), and the comprehensions of the ontology of the human being in universe that the performance work makes palpable in movement.

Within the dance field, the blurry unification of art/ science challenges choreographers, dancers, and audiences to reimagine each other. McGregor, Stewart, Stapiello and Jones create dance and in so doing capture an aesthetic that is changing (Oikonomou, 2012) where the role of environment is alive and interactional, and the role of creator is across the witnessing and doing of the moment of the performance. Dance-artists in 21st Century are exploring the boundaries of new technologies, manifesting hybrid art forms that are wider, richer and more diverse (Brooks, 2008). The choreographer moves down a path that forces critics, scholars and dancers, themselves, to redefine their understanding of the art form. A place where the sciences, the arts and the humanities share a common space of exchange offers the possibility of encounters with each other that allow us to be reflective, hopeful and open to what we can create together. The global collaboration of how we move in new technology offers us the opportunity to dance together, to explore how 'I' can end and begin in the global 'we' that is the 21 st century.

\section{References}

Akinleye A. (2019). “...wind in my hair, I feel a part of everywhere...": creating dance for young audiences narrates emplacement, Journal of Dance and Somatic Practices, vol. 11, pp. 39-47
Australian Dance Theatre (2019). Research. Retrieved from: https://www.adt.org.au/research

Boenisch P. M. (2006). Mediation Unfinished: Choreographing Intermediality in Contemporary Dance Performance, Rodopi, pp. 151-166 Brooks, P. (2008). Remediation of Moving Bodies: Aesthetic Perceptions of a Live, Digitised and Animated Dance Performance, Cultural Studies Journal of Universitat Jaume I, vol. VI, pp. 85-99.

Brown, C. (2007). Chance and circumstance: twenty years with Cage and Cunningham. New York: Alfred A. Knopf.

Buchanan, I. (2010). A Dictionary of Critical Theory. Oxford: Oxford University Press.

Corazza, G. E. (2016). Potential Originality and Effectiveness: The Dynamic Definition of Creativity, Creativity Research Journal, vol. 28, pp. 258-267

Cross, E. S., Ticini, L. F. (2011). Neuroaesthetics and beyond: new horizons in applying the science of the brain to the art of dance, Phenomenology and the Cognitive Sciences, vol. 11, $\mathrm{n}^{\circ}$ 1, pp. 5-16.

Cunningham, M., Monk, M., Jones, B. T. (1998). Art performs life : Merce Cunningham, Meredith Monk, Bill T. Jones. New York: Walker Art Center

Deleuze, G, Guattari, F. L. (1983). Anti-Oedipus: capitalism and schizophrenia. London: Athlone

Deleuze, G, Guattari, F. L. (1987). A thousand plateaus: capitalism and schizophrenia.

London: Athlone

Dewey, J. (1958). Experience and nature, New York:Dover Publications

Dewey, J. \& Boydston, J. A. (2008). The later works of John Dewey, 1925-1953. Carbondale: Southern Illinois University Press.

Dixon, S. (2007). Digital Performance: A History of New Media in Theater, Dance, Performance Art and Installation, Cambridge, MIT Press

Digfelder, S. F. (2010). Dance, dance, evolution. American Psychology Association vol. 40, $\mathrm{n}^{\circ}$ 4,

Ebenreuter, N. (2005). Dance Movement: A Focus on the Technology, IEEE Computer Society 
Farman, J. (2014). The Mobile Story: Narrative Practices with Locative Technologies. New York: Routledge

Franses, P. H. (2016). When Did Classic Composers Make Their Best Work?. Creativity Research Journal, vol. 28, pp. 219-221

Harrington, D. M. (2018). On the Usefulness of "Value" in the Definition of Creativity: A Commentary. Creativity Research Journal, vol. 30, pp. 118-121

Humphrey, D. (1965). El arte de crear danzas. Buenos Aires: Editorial Universitaria de Buenos Aires

Lindauer, M. S. (1998). Interdisciplinarity, the Psychology of Art, and Creativity; An Introduction. Creativity Research Journal vol. 11, pp. $1-10$

Long, H. (2014). An Empirical Review of Research Methodologies and Methods in Creativity Studies (2003-2012). Creativity Research Journal, vol. 26, pp. $427-438$

Studio Wayne McGregor (1992). Research. Retrieved from: http://waynemcgregor.com/research/ [accessed 10.05.18]

Meador S. W., Rogers T., O'Neal K., Kurt E., Cunningham C. (2004). Mixing Dance Realities: Collaborative Development of Live- Motion Capture in a Performing Arts Environment, ACM Computers in Entertainment, vol. 2

Merlau-Ponty, M. (2002). Phenomenology of perception, London: Routledge

Miller, A. I. (1984). Imaginery in Scientific Thought. Boston: Birkhauser

Muntanyola, D. (2014). How Multimodality shapes Creative Choice in Dance. Revista Internacional de Sociología, vol. 72, n 3, pp. 563-582.

New York Live Arts (2019). About the company. Retrieved from: https://newyorklivearts.org/btjaz-company/about-thecompany/

Oikonomou, N. (2012). Dance Research, Choreography and Cognitive Science: The encounter with the Creative Process-Analysis and Proposals for support of the Creative Choreographic Process in Dance (Bachelor Dissertation). University of the Aegean, Syros
Respini, E., Sulcas, R., Neri, L. (2018). William Forsythe : Choreographic objects, Boston:

The Institute of Contemporary Art

Root-Bernstein, R. S. (1989). Discovering: Inventing and Solving Problems at the Frontiers of Scientific Knowledge, Cambridge: Harvard University Press

Runco M., Jaeger G. (2012). The Standard Definition of Creativity. Creativity Research Journal, vol. 24, pp. 92-96

Pérez-Fabello, M., Campos A. (2007). Influence of Training in Artistic Skills on Mental Imaging Capacity. Creativity Research Journal, vol. 19, pp. 227-232

Preciado-Azanza, G. (2018). Professional Inquiry: Understading the impact of new technologies on the creative process in the $21^{\text {st }}$ Century Professional Dance (Bachelor Dissertation). Middlesex University, London

Preciado-Azanza, G. (2014). How has Wayne Mcgregor used Science to develop his choreography in the $21^{\text {st }}$ century dance? (Diploma Dissertation). English National Ballet School, London

Salter, C. (2010). Entangled: Technology and the Transformation of Performance. Cambridge: The MIT Press

Sparacino F., Davenport G., Pentland A. (2000). Media in performance: Interactive spaces for dance, theatre, circus and museum exhibits. IBM Systems Journal, vol. 39, pp. 479-510

Sternberg, R. J. (2006). The Nature of Creativity. Creativity Research Journal, vol. 18, pp. 87-98

Tornero, P. (2013). Tecnologías de la Creatividad: Conexiones entre Arte y Ciencia en la Contemporaneidad (Doctoral Dissertation). Universidad Complutense de Madrid, Madrid

Troika Ranch (2019). Works. Retrieved Available from: http://troikaranch.org/\#section-works

Vincs K, McCormick J. (2010). Touching Space: Using Motion Capture and Stereo Projection to Create a Virtual Haptics of Dance. Leonardo, vol. 43, pp. 359-366

Vincs K, Barbour K. (2014). Snapshots of complexity: using motion capture and principal component 
analysis to reconceptualise dance. Journal Digital Creativity, vol. 25, pp. 62-78

World Dance Council Education Department (2012). Creativity by David Kirsch. Retrieved from http:// wdced.com/2012/02/creativity-david-kirsh/ [accessed 03.01.19]

Wilson, S. (2010). Art + Science Now: How scientific research and technological innovation are becoming key to 21st-century aesthetics. New York: Thames \& Hudson
Wynter, S. (2003). Unsettling the Coloniality of Being/ Power/Truth/Freedom: Towards the

Human, After Man, Its Overrepresentation - An Argument. CR: The New Centennial Review, vol. 3, pp. 257-337. 